\title{
CORRESPONDENCE
}

\section{An unusual case of hypoplastic internal jugular vein in a vein of Galen malformation}

\author{
Prabhakar S. Prakash, Virendra Jain, \\ Kavita Sandhu ${ }^{1}$
}

Vein of Galen malformations (VOGMs) are rare anomalies of intracranial circulation that constitute 1\% of all intracranial vascular malformations. However, they represent $30 \%$ of vascular malformations presenting in the paediatric age group. ${ }^{[1]}$ These lesions are characterised by the presence of an aneurysmally dilated midline deep venous structure, fed by abnormal arteriovenous communications. The venous system of the brain is mal-developed many venous anomalies can associate with VOGM. We describe a case of VOGM in which there was hypoplasia of the right internal jugular vein (IJV) leading to failure in the placement of central venous catheter through right IJV.

A 7-year-old child presented with peri-orbital swelling and proptosis (left more than right). In the history, 3 years ago, the patient had suffered an episode of hemiparesis for approximately 15 days which resolved completely without any residual deficit. On examination, the swelling seemed to be vascular in nature. A provisional diagnosis of an arteriovenous malformation (AVM) was made. A digital subtraction angiography (DSA) was planned under general anaesthesia. General anaesthesia was instituted as per standard protocol with a $22 \mathrm{~g}$ intravenous cannula. Since the venous access was difficult, we decided to secure the central venous catheter. Initially, we tried as per standard practice. Even after three attempts, we are unable to locate the right IJV. Thereafter,

Department of Anaesthesia and Critical Care, Fortis Memorial Research Institute, Gurgaon, Haryana, ${ }^{1}$ Department of Neuroanaesthesiology and Critical Care, Institute of Neurosciences, Max Super Speciality Hospital, Saket, New Delhi, India

Address for correspondence:

Dr. Prabhakar Suman Prakash, Department of Neuroanaesthesiology and Critical Care, Institute of Neurosciences, Max Super Speciality Hospital, 1, Press Enclave Road, Saket, New Delhi - 110 017, India. E-mail: prabhakarsuman73@yahoo.co.in we attempted an ultrasonography (USG) guided IJV placement. The right IJV could not be visualised even with the USG. Thereafter, we successfully placed catheter in the right subclavian vein. On DSA, the patient was found to have a choroidal type of VOGM. There was absence of straight sinus with the fistula draining via a prominent falcine sinus. Both the transverse sinuses, sigmoid sinuses, jugular bulbs and IJVs were not opacified in any of the injections. There was venous rerouting to bilateral, cavernous sinuses (left was dominant) and then into the facial and intra-spinal veins [Figures 1 and 2].

VOGMs are associated with several arterial and venous anomalies. In most cases, these anomalies present in

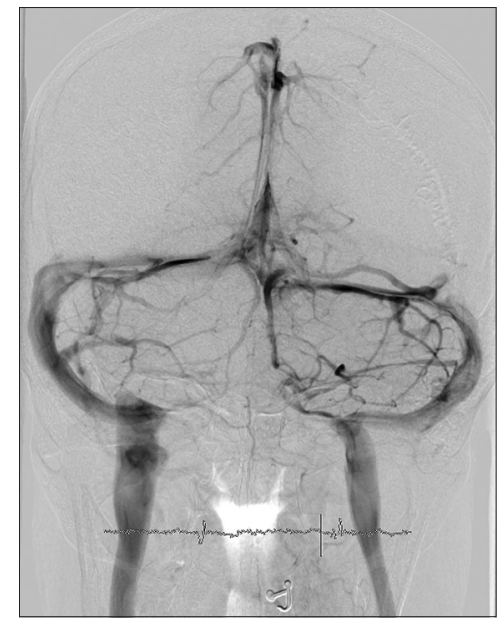

Figure 1: Digital subtraction angiography of brain showing normal venous drainage (another patient)

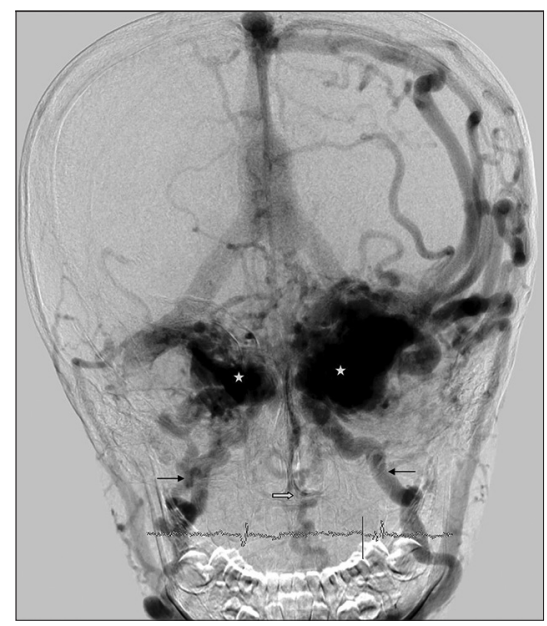

Figure 2: Digital subtraction angiography of the brain, antero-posterior view, venous phase, showing abnormal venous drainage pattern with non-visualisation of transverse and sigmoid sinuses as well as jugular bulb and internal jugular veins. The venous drainage is into both ophthalmic and then facial veins (small black arrows) via cavernous sinuses (stars) as well as the intra-spinal veins (block arrow) 
the neonatal period or early childhood. Depending on the size of the shunt, adequacy of venous drainage, complexity of arterial supply and the host response, the cardiac manifestations can range from asymptomatic cardiomegaly to severe cardiac failure that is, refractory to medical management. Older children and adults usually have low flow fistulae presenting with headache and seizures. A small number of patients may also present with developmental delay, focal neurological deficits, proptosis and epistaxis. ${ }^{[2]}$ In our case, the presentation was proptosis and periorbital swelling.

Although ultrasound guidance is the current recommendation for central line placement in IJV, conventional practice is still followed due to unavailability of the machine or lack of expertise. In our case, we tried ultrasound to locate the IJV but failed to find it, and it was only after DSA that it confirmed that the vein was hypoplastic. Venous anomaly can present in any patient, but we should entertain a high degree of suspicion in the case of AVM, especially VOGM. Catheter placement with ultrasound guided practice is definitely a good modality to overcome such pitfalls.

\section{Financial support and sponsorship} Nil.

\section{Conflicts of interest}

There are no conflicts of interest.

\section{REFERENCES}

1. Bhattacharya JJ, Thammaroj J. Vein of Galen malformations. J Neurol Neurosurg Psychiatry 2003;74 Suppl 1:i42-4.

2. Gupta AK, Varma DR. Vein of Galen malformations: Review. Neurol India 2004;52:43-53.

This is an open access article distributed under the terms of the Creative Commons Attribution-NonCommercial-ShareAlike 3.0 License, which allows others to remix, tweak, and build upon the work non-commercially, as long as the author is credited and the new creations are licensed under the identical terms.

\begin{tabular}{|l|l|}
\hline \multicolumn{2}{|c|}{ Access this article online } \\
\hline Quick Response Code: & Website: \\
\hline & www.jnaccjournal.org \\
\cline { 2 - 2 } & \\
\hline
\end{tabular}

